\title{
Serum Metabolomics Study Based on LC-MS and Antihypertensive Effect of Uncaria on Spontaneously Hypertensive Rats
}

\author{
Ana Liu, ${ }^{1}$ Yan-Jun Chu, ${ }^{2}$ Xiaoming Wang, ${ }^{1}$ Ruixue Yu, ${ }^{1}$ \\ Haiqiang Jiang $\mathbb{D},{ }^{3}$ Yunlun Li $\mathbb{D},{ }^{2}$ Honglei Zhou, ${ }^{1}$ Li-Li Gong, ${ }^{3}$ \\ Wen-Qing Yang, ${ }^{2}$ and Jianqing $\mathrm{Ju}^{2}$ \\ ${ }^{1}$ Pharmacy School, Shandong University of Traditional Chinese Medicine, Jinan 250355, China \\ ${ }^{2}$ Traditional Chinese Medicine Clinical Research Base for Hypertension of Affiliated Hospital, Shandong University of \\ Traditional Chinese Medicine, Jinan 250011, China \\ ${ }^{3}$ Experimental Center, Shandong University of Traditional Chinese Medicine, Jinan 250355, China
}

Correspondence should be addressed to Haiqiang Jiang; jhq12723@163.com and Yunlun Li; yunlun.lee@hotmail.com

Received 13 July 2017; Revised 15 October 2017; Accepted 26 November 2017; Published 4 April 2018

Academic Editor: Bhavanath Jha

Copyright (c) 2018 Ana Liu et al. This is an open access article distributed under the Creative Commons Attribution License, which permits unrestricted use, distribution, and reproduction in any medium, provided the original work is properly cited.

\begin{abstract}
Our previous studies have shown that Uncaria has an important role in lowering blood pressure, but its intervention mechanism has not been clarified completely in the metabolic level. Therefore, in this study, a combination method of HPLC-TOF/MS-based metabolomics and multivariate statistical analyses was employed to explore the mechanism and evaluate the antihypertensive effect of Uncaria. Serum samples were analyzed and identified by HPLC-TOF/MS, while the acquired data was further processed by partial least squares discriminant analysis (PLS-DA) and orthogonal partial least squares discriminant analysis (OPLS-DA) to discover the perturbed metabolites. A clear cluster among the different groups was obtained, and 7 significantly changed potential biomarkers were screened out. These biomarkers were mainly associated with lipid metabolism (dihydroceramide, ceramide, PC, LysoPC, and TXA2) and vitamin and amino acids metabolism (nicotinamide riboside, 5-HTP). The result indicated that Uncaria could decrease the blood pressure effectively, partially by regulating the above biomarkers and metabolic pathways. Analyzing and verifying the specific biomarkers, further understanding of the therapeutic mechanism and antihypertensive effect of Uncaria was acquired. Metabolomics provided a new insight into estimate of the therapeutic effect and dissection of the potential mechanisms of traditional Chinese medicine (TCM) in treating hypertension.
\end{abstract}

\section{Introduction}

Hypertension, a complex and multifactorial disease, is closely associated with gene, environment, and metabolic factor, also the most vital risk factor for cardiovascular disease and chronic kidney disease [1]. With the development of study, various theories had been proposed on the pathogenesis of hypertension. Previous research had indicated that endothelial dysfunction might be a crucial mechanism in pathogenesis of hypertension [2]. Under the damage of endothelial cells, active molecules such as ATP and 5-HT would be released. Because these molecules inhibited the activities of NO, CGRP, and other relaxing factors, the activities of ET and
TXA2 were increased. Finally, these substances would lead to the increasing blood pressure level [3-5]. In another study, hypertension was considered as a neurogenic disease and it is proposed that the pathology of hypertension was significantly associated with multiple factors in neuroendocrine system [6].

In modern medicine, the hypertension had been studied from above perspectives. In the treatment of hypertension, the traditional Chinese medicine (TCM) has formed a unique theoretical system and control methods. Chinese herbal medicines provided rational means for hypertension. Uncaria was employed in treating hypertension as a safe and effective therapy due to characteristics of multiple targets, 
multiple pathways, and fewer side effects [7]. Uncaria, with Chinese name of Gouteng and the Latin name of Uncaria rhynchophylla (Miq.) from Chinese Pharmacopoeia 2015 version, was firstly recorded in the Chinese medical classics "Compendium of Materia Medica." Rhynchophylline and isorhynchophylline are the mainly bioactive components in Uncaria $[8,9]$. Our previous studies indicated that Uncaria had effects on lowering blood pressure, protecting vascular endothelial cell, and inhibiting vascular smooth muscle proliferation $[10,11]$. The antihypertensive mechanism of Uncaria is still unclear, although it has been in widespread application. In modern medicine, the holistic and systemic properties of TCM are consistent with the characters of metabolomics. Therefore, metabolomics might be the bridge between TCM and modern medicine. So metabolomics could be employed to perform such a comprehensive investigation.

Metabolomics, as an important branch of systems biology, provides a comprehensive insight into the rule of metabolite changes. The metabolic variations of organism could be able to be systematically explored with the comprehensive analysis of metabolic result [12]. At present, metabolomics has been widely used in animal model validation [13], disease diagnosis [14], drug toxicity evaluation [15], and drug research and development [16]. And it could evaluate and interpret therapeutic effects of some Chinese medicines [17-19]. Metabolomics analysis required highdensity information on a wide range of compound classes and chemistries, as well as high precision. Many technologies, including NMR, GC-MS, LC-MS, and CE-MS [20], have been developed to perform metabolomics analysis. Because of its high sensitivity, high precision, low sample size, and good reproducibility, LC-MS became one of the most frequent significant tools in metabolomics researches [21].

In this study, the metabolic profiling in serums of spontaneously hypertensive rats (SHRs) and drug treated SHRs was analyzed by HPLC-MS. Both antihypertensive efficacy and potential mechanism of Uncaria were explored in our experiment. The results of this paper provided further evidence to understand the pathology of hypertension and the intervention effects of Uncaria.

\section{Materials and Methods}

2.1. Chemicals and Reagents. The dried Uncaria (collected in Huaihua, Hunan, China) was authenticated as stem and branch with hook from Uncaria rhynchophylla (Miq.) by Professor Feng Li (School of Pharmacy, Shandong University of Traditional Chinese Medicine). The content of Uncaria rhynchophylline (main active component in Uncaria) was $5.117 \%$, which was determined by HPLC-QQQ/MS (Agilent Technologies, USA). More detailed information was displayed in the Supplementary Materials: MRM parameters and MRM chromatograms of four alkaloids were shown in the Figure a and Table a. The linear equation, linear range, correlation coefficient, quantitative limits, and detection limits of four alkaloids in Uncaria were described in Table b. Table c showed the contents of four alkaloids in the Uncaria extract used in this experiment. The methodological results of precision, stability, repeatability, and recovery in the determination of Uncaria content were also illustrated in the supplementary material. Acetonitrile of HPLC grade was purchased from Merck (Darmstadt, Germany). Formic acid of HPLC grade was obtained from Tedia (Fairfield, $\mathrm{OH}, \mathrm{USA}$ ). Distilled water was produced by Milli-Q Reagent water system (Millipore, MA, USA). Others were of analytical grade.

2.2. Preparation of Uncaria and Chemical Profile. Powdered Uncaria was extracted with $95 \%$ ethanol (10 times, for three times) under thermal reflux for 1 hour. The resulting residue was extracted with water (for three times) with the same condition as ethanol. After filtration, the ethanol and water extracts were combined and concentrated under reduced pressure at $60^{\circ} \mathrm{C}$. Finally, the concentration of the stock solution of Uncaria extract was adjusted to $1.0 \mathrm{~g} / \mathrm{crude}$ herb $/ \mathrm{mL}$ and stored at $4^{\circ} \mathrm{C}$.

2.3. Animals and Groups. All animals were purchased from the Vital River Laboratory Animal Technology Co. Ltd. (Beijing, China), certificate number SCXK (Beijing) 2012-0001. All studies were carried out by following the guidelines of National Research Council of China and approved by Animal Ethics Committee of Shandong University of Traditional Chinese Medicine. A total of 16 male SHRs (age 12 weeks) were divided randomly into model group $(n=8)$ and an Uncariatreated group $(n=8)$. Another $8 \mathrm{Wistar}$ rats were involved as the normal group. All rats were bred under standard animal conditions with regulated temperature $\left(17-25^{\circ} \mathrm{C}\right)$, humidity (45-60\%), and $12 \mathrm{~h} / 12 \mathrm{~h}$ light/dark cycle; they were admitted to free access to food and water during the study period. In the Uncaria-treated group, rats were given Uncaria (the dose was $10.001 \mathrm{~g}$ raw materials/Kg SHRs). The normal group and model group were given equal quantities of physiological saline. In accordance with the principle of the same volume and the different concentration, all animals were given gastric infusion of one time each day for continuous 4 weeks. One rat was died in Uncaria group at the end of experiment. Finally, 23 rats were included in the later experimental analysis.

2.4. Blood Pressure Measurement. Blood pressure of rats was measured on tail artery by noninvasive blood pressure measurement before administration and after administration for 1 week, 2 weeks, 3 weeks, and 4 weeks. Acquired data was expressed by means $\pm \mathrm{SD}$, and the multigroup comparisons were analyzed by one-way analysis of variance (ANOVA) using SPSS 22.0 (SPSS Inc., USA).

\subsection{Collection and Preparation of Serum Samples}

2.5.1. Serum Sample Preparation. Serum was collected from the abdominal aorta and immediately centrifuged at 4,000 rpm for $15 \mathrm{~min}$. The supernatant was transferred into a clean tube and stored at $-80^{\circ} \mathrm{C}$. Prior to analysis, $300 \mu \mathrm{L}$ serum was added with $600 \mu \mathrm{L}$ acetonitrile and vortexed for $1 \mathrm{~min}$ to precipitate the proteins. Then the samples were 
centrifuged at 12,000 rpm for $15 \mathrm{~min}$ and transferred to vials for LC/MS analysis.

2.5.2. Quality Control Sample Preparation. An amount of $40 \mu \mathrm{L}$ of serum from each rat was mixed to serve as a quality control (QC) sample, which was pretreated as described in Section 2.5.1. QC sample was applied for monitoring the repeatability of samples and validating the stability of HPLCMS during the analysis. As the sequence running, the QC sample was analyzed six times in the beginning and randomly arranged after 6 unknown serum samples.

2.6. HPLC-MS Conditions. HPLC-MS analysis was performed on Agilent 6230 HPLC-TOF system (Agilent Technologies, USA). Chromatographic separation was performed on a Halo- $\mathrm{C}_{18}$ column $(2.1 \times 100 \mathrm{~mm}, 2.7 \mu \mathrm{m}$, America Advanced Material Technology Cor.) with a binary solvents system (solvent A: water with $0.1 \%$ formic acid; solvent B: acetonitrile with $0.1 \%$ formic acid). The gradient elution program for analysis started from $10 \%$ B to $60 \% \mathrm{~B}$ at $0-15 \mathrm{~min}$, was held at $60 \%$ for $7 \mathrm{~min}$, and changed to $100 \% \mathrm{~B}$ over $22-24 \mathrm{~min}$. The flow rate was $0.35 \mathrm{~mL} \cdot \mathrm{min}^{-1}$ and the injection volume was $5 \mu \mathrm{L}$. The column temperature was set at $25^{\circ} \mathrm{C}$.

MS Conditions. They are as follows: capillary voltage, $4.0 \mathrm{kV}$; drying gas flow, $11 \mathrm{~L} \cdot \mathrm{min}^{-1}$; gas temperature, $350^{\circ} \mathrm{C}$; nebulizer pressure, $35 \mathrm{psig}$; fragmentor voltage, $140 \mathrm{~V}$; skimmer voltage, $60 \mathrm{~V}$. Data was collected in centroid mode from 100 to $1,000 \mathrm{~m} / z$. Samples were analyzed randomly for unbiased measurement with reference solution $(\mathrm{m} / z 121.050873$ and 922.009798) as internal standards to ensure accuracy and reproducibility. Two different fragmentor voltages at 210 and $280 \mathrm{~V}$ were applied to search the target ion of biomarkers.

2.7. Multivariate Data Analysis and Data Processing. The raw data were firstly converted to.cef format by Mass Hunter Qualitative Analysis Software (Agilent Technologies, USA) and imported into Mass Profiler Professional (MPP, Agilent Technologies, USA) to carry on preliminary calibration of retention time, abundance, and mass range. The data from each sample were normalized to the summed total area and all data were input into Mass Hunter secondly to execute regression analysis. Afterwards data were processed by MPP again to carry on filter flags and significance analysis. Finally, a three-dimensional data matrix which consisted of retention time, mass value, and peak intensity was built. Then the data matrix was imported into SIMCA-P (version 11.5, Umetrics) software in ".csv" format for multivariate statistical analysis; PLS-DA and OPLS-DA were performed for pattern recognition. Variable importance projection (VIP) produced by OPLS-DA, ANOVA, and fold change (FC) was applied to discover the contributable-variable for classification. Finally, the variables with VIP values $>1, p$ value $<0.05$, and FC value $\geqslant 2$ were treated as potential biomarkers. The exact mass of potential biomarkers was searched in database such as HMDB (http://www.hmdb.ca), METLIN (https://metlin .scripps.edu), and KEGG (http://www.genome.jp/kegg/) for biomarkers identification.

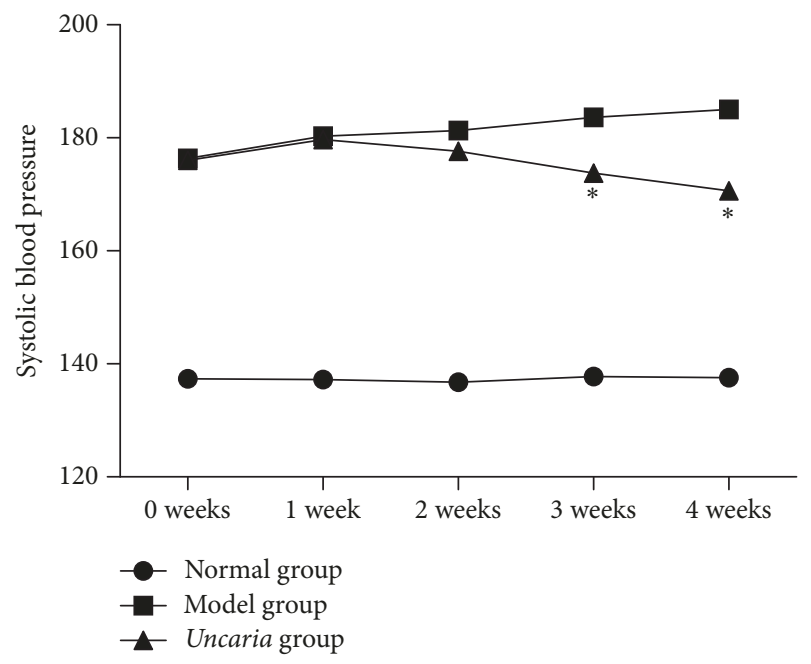

FIGURE 1: Systolic blood pressure changes in 4-week consecutive administration $(\mathrm{mmHg})$. Note. ${ }^{*} p<0.05$ compared with model group.

\section{Results}

3.1. Blood Pressure. Compared with the normal group, the systolic blood pressure (SBP) in the model group was significantly elevated and maintained stable during the drug intervention. Meanwhile, with the treatment of Uncaria for 3 weeks, the SBP in Uncaria-treated group was lower than model group $(p<0.05)$. With the prolonging of administration time, SBP of Uncaria-treated group declined continuously and reached a minimum at the end of the 4th week. These data demonstrated that Uncaria had an obvious effect on decreasing blood pressure. The results are shown in Figure 1.

3.2. HPLC-MS Analysis of Serum. Different kinds of endogenous metabolites could be simultaneously detected with HPLC-MS under the optimized conditions. The extract ion chromatogram (EIC) of serum samples of seven biomarkers from Wistars, SHRs, and Uncaria-treated SHRs were presented in Figure 2. It showed the differences of EIC in samples from normal group, model group, and Uncaria-treated group.

3.3. Data Quality Evaluation. The experimental data of QC samples were imported into SIMCA-P for principal component analysis (PCA). The results showed that the clustering of QC data was closed and concentrated in the $95 \%$ confidence interval. This indicated that the data quality was reliable, but the results were not displayed in this paper. In order to validate the reliability of data quality further, 10 ions (the ion's quality number from low to high) were selected from QC samples with the retention time and $\mathrm{m} / z$ pairs of 0.74-113.0909, 0.65-176.0316, 0.64-250.1682, 0.57-363.9451, $17.40-426.3592,21.51-551.3353,27.34-676.4716,8.65-736.1158$, 21.54-850.5669, and 17.97-991.6764. The RSDs of peak area were within the range of $0-0.22 \%, 0-0.60 \%$, and $0-0.50 \%$ in three groups; this indicated that the data quality is reliable. 


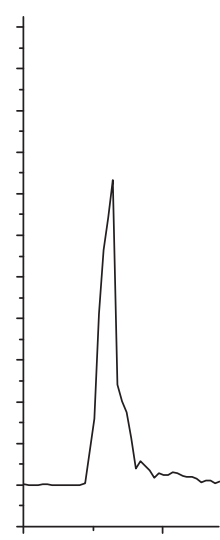

Dihydroceramide

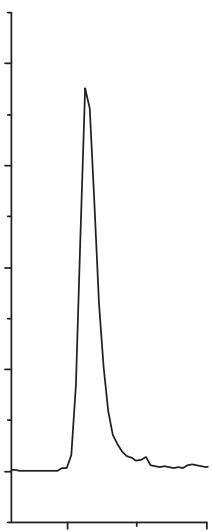

Dihydroceramide

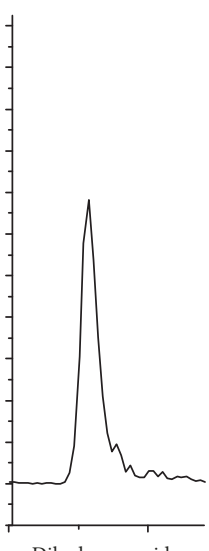

Dihydroceramide

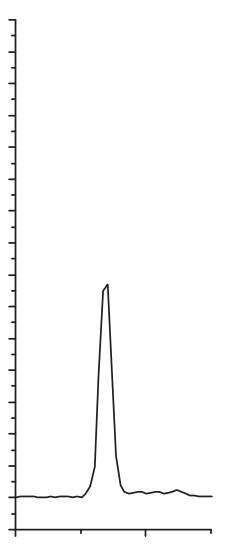

Ceramide
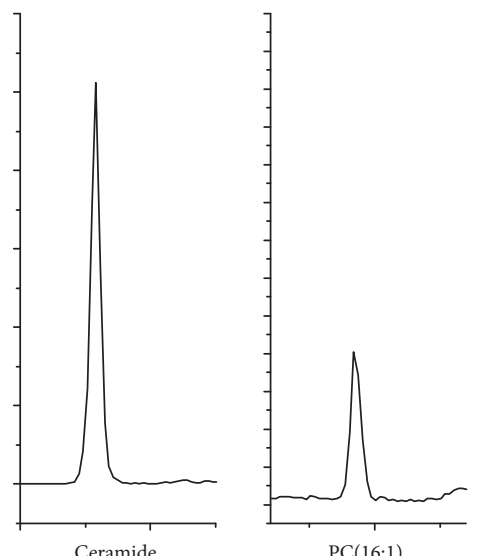

$\operatorname{PC}(16: 1)$
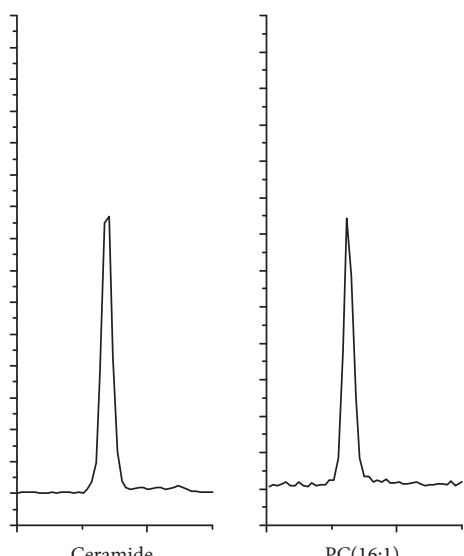

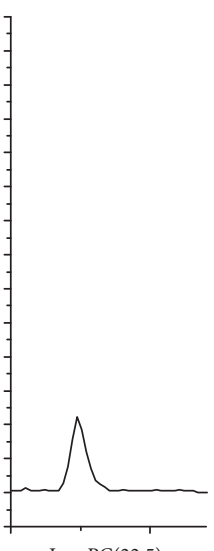

ysoPC $(22: 5)$

(a)

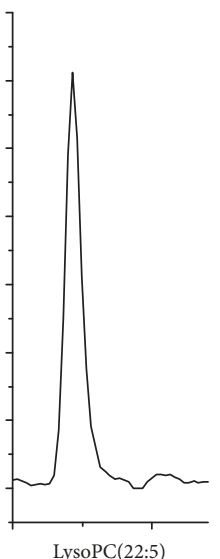

(b)

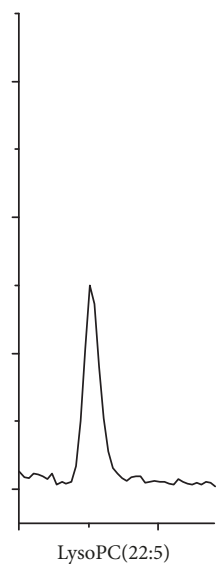

(c)
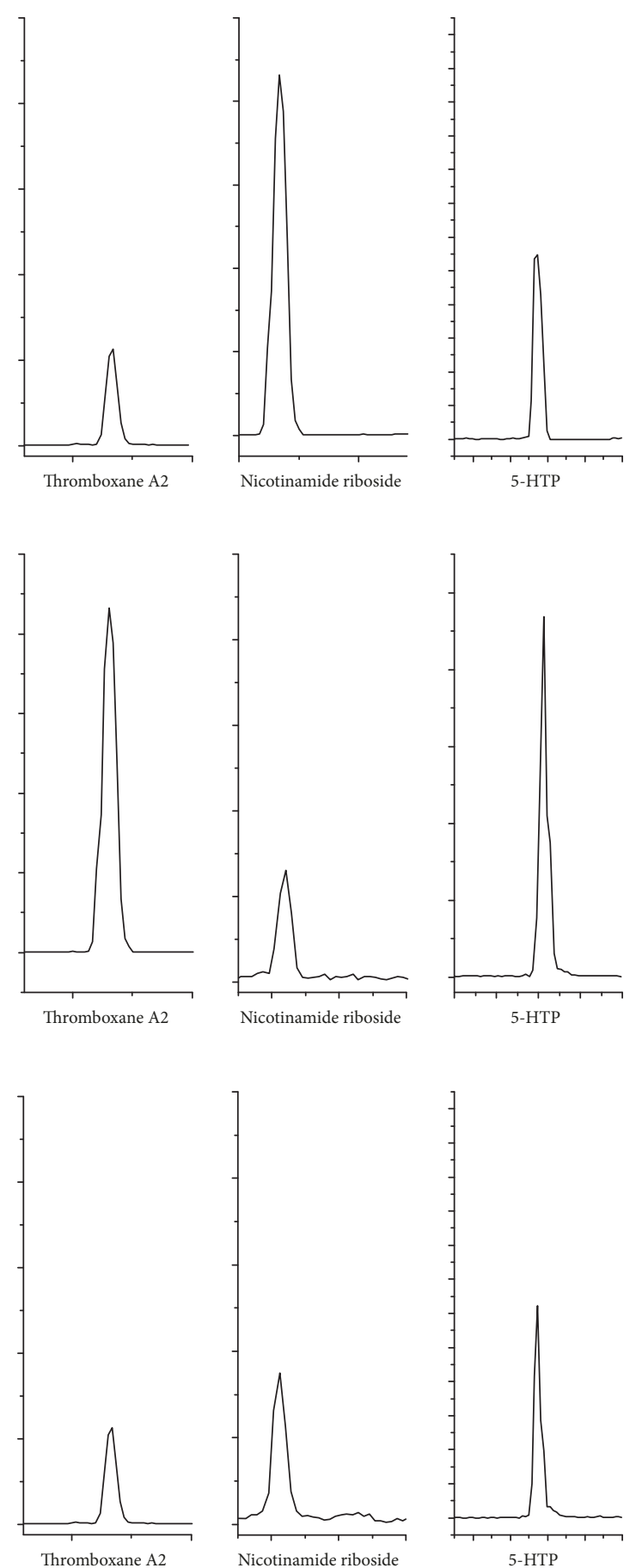

5-HTP

FiguRE 2: HPLC-MS extract ion chromatogram (EIC) of serum samples from the normal group (a), the model group (b), and the Uncariatreated group (c).

3.4. Multivariate Data Analysis. In this study, ion regression was applied to improve the accuracy of the MFE lookup. A calibrated and filtered “.cef” file was input into Mass Hunter secondly to executing regression analysis. The comparison of results before and after ion regression showed that compounds that appeared only 1 time would be significantly reduced after regression, and most compounds appeared in all 23 samples (Figure 3). The results indicated that both the feature search and calibration parameters were successful.

PLS-DA is a supervised method for pattern recognition. In PLS-DA score plot (see Figure 4(a)), there was a clear separation in space between the normal and model group, 
TABLE 1: Potential biomarkers in samples and corresponding metabolic pathways.

\begin{tabular}{|c|c|c|c|c|c|c|c|}
\hline \multirow{2}{*}{ Number } & \multirow{2}{*}{ RT } & \multirow{2}{*}{ Mass } & \multirow{2}{*}{$\begin{array}{l}\text { Biomarker } \\
\text { identification }\end{array}$} & \multirow{2}{*}{ KEGG } & \multicolumn{2}{|c|}{ Change trend } & \multirow{2}{*}{ Pathway } \\
\hline & & & & & Trend $^{\mathrm{a}}$ & Trend $^{\mathrm{b}}$ & \\
\hline (1) & 15.64 & 329.3297 & Dihydroceramide & $\mathrm{C} 12126$ & $\uparrow$ & $\downarrow$ & Sphingolipid metabolism \\
\hline (2) & 28.35 & 563.441 & Ceramide & C00195 & $\uparrow$ & $\downarrow$ & Sphingolipid metabolism \\
\hline (3) & 29.94 & 701.2065 & PC(16:1(9Z)/14:1(9Z)) & C00416 & $\downarrow$ & $\uparrow$ & $\begin{array}{l}\text { Glycerophospholipid } \\
\text { metabolism }\end{array}$ \\
\hline (4) & 18.39 & 569.3487 & LysoPC (22:5) & $\mathrm{C} 04230$ & $\uparrow$ & $\downarrow$ & $\begin{array}{l}\text { Glycerophospholipid } \\
\text { metabolism }\end{array}$ \\
\hline (5) & 26.68 & 296.2828 & Thromboxane A2 & C02198 & $\uparrow$ & $\downarrow$ & $\begin{array}{l}\text { Arachidonic acid } \\
\text { metabolism }\end{array}$ \\
\hline (6) & 26.68 & 255.2564 & Nicotinamide riboside & $\mathrm{C} 03150$ & $\downarrow$ & $\uparrow$ & $\begin{array}{l}\text { Nicotinate and nicotinamide } \\
\text { metabolism }\end{array}$ \\
\hline$(7)$ & 0.68 & 220.1793 & 5-HTP & $\mathrm{C} 00643$ & $\uparrow$ & $\downarrow$ & Tryptophan metabolism \\
\hline
\end{tabular}

Note. ${ }^{\text {a }}$ Trends of model group compared with normal group of metabolites. $p<0.05$ compared to normal group. ${ }^{\mathrm{b}}$ Trends of Uncaria group compared with model group of metabolites. $p<0.05$ compared to model group. $\uparrow:$ upregulated. $\downarrow$ : downregulated.

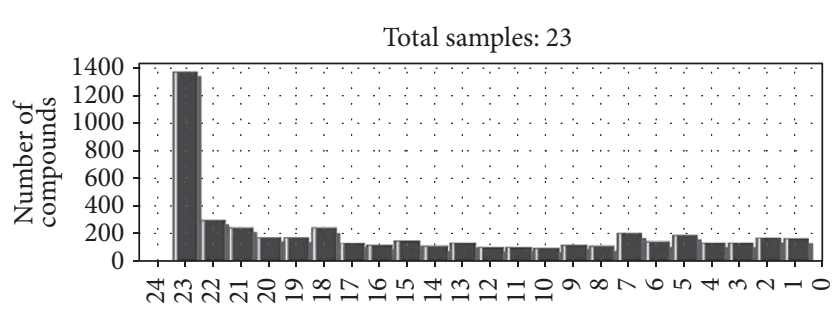

Frequency

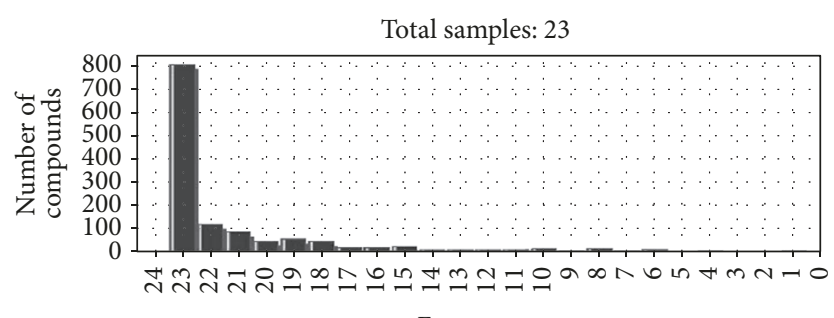

Frequency

Figure 3: The comparison of results before and after ion regression analysis.

as well as between Uncaria-treated group and model group. The results implied that the serum metabolic patterns were changed between the three groups. The Uncaria-treated group was much closer to the normal group, which indicated that Uncaria did have a potential antihypertensive effect on the SHRs. In addition, the PLS-DA model also enjoyed a good predictive ability and credibility: $R 2 Y=0.98$; $Q 2$ (cum) $=$ 0.83 . No overfitting was observed according to the results of permutation test. As shown in Figure 4(b), the $R^{2} Y$-intercept was 0.306 and $Q^{2}$ was -0.486 in the positive ion modes, respectively. Furthermore, all green $R 2$-values to the left were lower than the original points to the right, indicating that the original model was valid.

3.5. Potential Biomarkers Discovery and Identification. The OPLS-DA was utilized to study the blood samples of the Uncaria group and the model group. The model evaluation index of $R^{2} Y$ and $Q^{2} Y$ was 0.994 and 0.924 , respectively, which indicated that the model had good prediction characteristics. As shown in the OPLS-DA score plot (Figure 5(a)), the Uncaria group and the model group can be clearly distinguished. More subtle changes can be found by the loading plot of OPLS-DA (Figure 5(b)), which exhibits each of these variables and is responsible for the separation more intuitively. For further analysis of feature ions, the S-plot (Figure 5(c)) and VIP-plot (Figure 5(d)) from the OPLSDA were carried out to select distinct variables as potential biomarkers. From the corresponding S-plot and VIP-plot, the ions furthest away from the origin may be regarded as potential biomarkers in Uncaria rats. We generated VIP plots from the OPLS-DA with a threshold of 1 to identify the metabolites that significantly contribute to the clustering between groups. Ultimately, 7 potential biomarkers were identified. By comparison with the normal group, 5 metabolites were increased in the model group, and the 2 metabolites were decreased. 7 metabolites tended to normal levels after intervention by Uncaria. The differences and change trend of these biomarkers are summarized in Table 1 and Figure 6.

\section{Discussion}

With the deepening research on the pathogenesis of hypertension, a variety of theories have been put forward. At present, the majority of studies suggest that vascular endothelial dysfunction is an important mechanism in the pathogenesis of hypertension [22]. The NO, CGRP, and other diastolic factors' activity declined and ET, TXA2, and other vasoconstrictor substances' secretion increased due to the vascular endothelial dysfunction, which can result in elevating blood pressure $[23,24]$. Therefore, the improvement of vascular endothelial function has important significance for the treatment of hypertension. At present, vascular endothelial function becomes a new target for antihypertensive therapy of TCM [25]. Previous studies illustrated that Uncaria extract can protect the structure and function of vascular endothelium by reducing vasoconstrictor release, increasing 


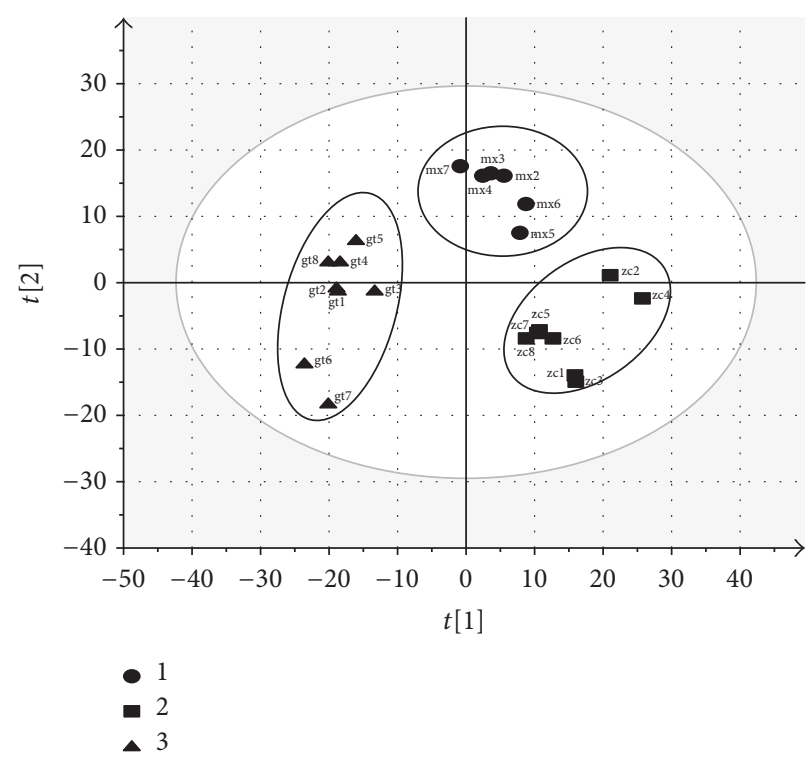

(a)

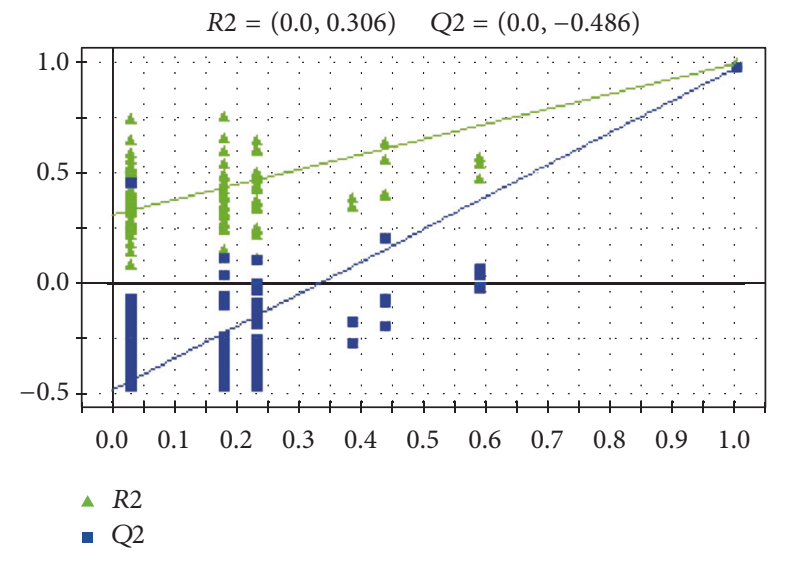

(b)

FIGURE 4: PLS-DA score plot (a) (1: model group; 2: normal group; 3: Uncaria group) and validated model plots (b) based on the HPLC-MS data.

the biological activity of $\mathrm{NO}$, and other ways. These ways can produce vasodilation effects; thereby, the blood pressure was reduced.

In this paper, a metabolomics study was conducted to analyze the changes of endogenous responses in SHRs after intervention with Uncaria. The results suggested that disturbed metabolic profiling was restored in normal level after the treatment of Uncaria. 7 biomarkers were identified, and their changes were associated with the hypotensive effect. The biomarkers were mainly involved in three biological pathways: lipid metabolism pathway, amino acid metabolism pathway, and nicotinic acid and nicotinamide metabolism pathway. The above biomarkers and corresponding pathways are associated with vascular endothelial dysfunction and other etiologies of hypertension. At the same time, we proposed a pathogenesis of hypertension associated with metabolic disturbance (Figure 7).

The substance in blue boxes represented biomarkers determined by HPLC-MS, and the pink boxes represented the pathways related to the hypertension.

4.1. Lipid Metabolism. The experimental result has revealed that the lipid metabolism disordered, which was consistent with previous conclusions [26]. There is widespread evidence that altering lipid metabolism is the potential pathogenesis of hypertension. Many studies have found abnormal phospholipid metabolism plays an essential role in development of intimal injury and vascular disease. Dyslipidemia may be relevant to the ox-LDL pathway, leading to a decrease of NO and PGI2, as well as the increase of ET-1 and TXA2; both of these effects bring a rise in blood pressure corporately.

A finding showed that sphingolipids were involved in the pathophysiology of hypertension in vivo [27]. Experimental study proved that PKC, Rac, Jun N-terminal kinase, and other protein kinases may be activated by ceramide; then cascade signaling pathways may be initiated to induce cell apoptosis [28]. Thereby, the activation of eNOS was inhibited, oxidative stress response of vascular endothelial cells was induced, and production of $\mathrm{NO}$ was decreased, finally leading to vascular endothelial dysfunction [29]. In SHR, the increase of TXA2 release which was induced by ceramide could be caused vasoconstriction, and the vascular constriction was also associated with increased expression of TXA2 synthase [30].

In our study, compared with the model group, dihydroceramide and ceramide were significantly decreased in Uncaria-treated group. Their changes were consistent with the antihypertension effect, and the interference of sphingolipid metabolism can be recovered after the administration of Uncaria.

By comparison with normal group, the level of TXA2 was upregulated in model group (Figure 6). TXA2 was produced by arachidonic acid in cyclooxygenase pathway, which was able to promote platelet aggregation and vascular contraction [31]. In the pathological process of hypertension, subendothelial collagen and fiber were exposed because vascular endothelium was damaged, combined with platelet to activate and release TXA2. It can increase the peripheral resistance by inhibiting the synthesis of PCI2, affecting the permeability of vascular wall, and promoting the proliferation of smooth muscle cells. Then the blood pressure would be persistently elevated $[32,33]$. Furthermore, Uncaria made effects on antihypertension through reducing TXA2 level. This result was consistent with the previous study of XIE [34]. Overall, aforementioned variations of lipids suggested lipid metabolism would be disturbed by hypertension, which could be partly regulated and repaired with Uncaria treatment. 


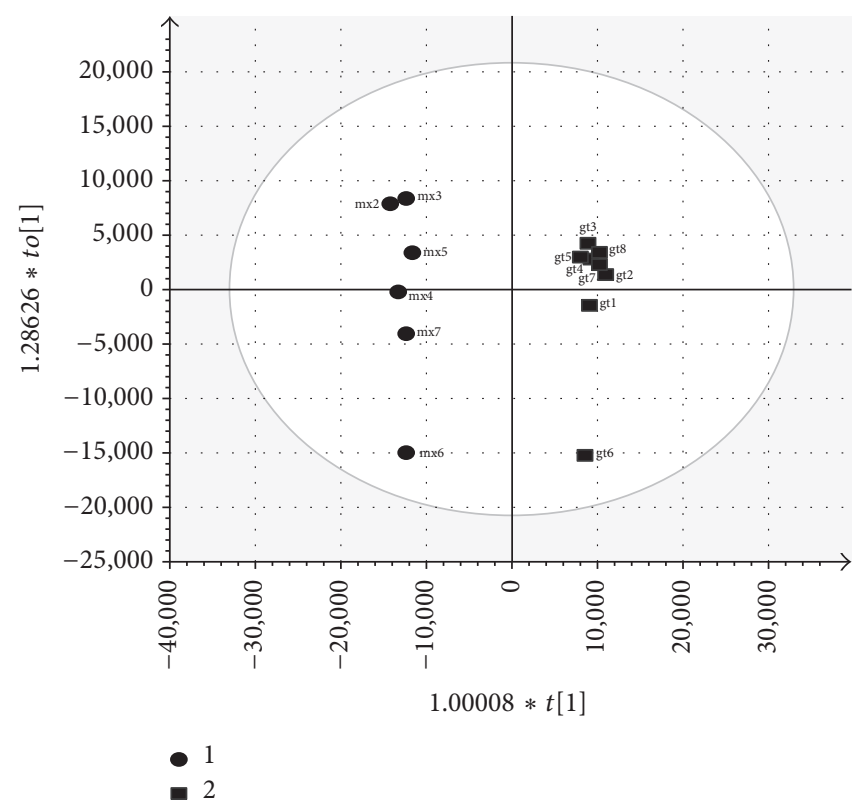

(a)

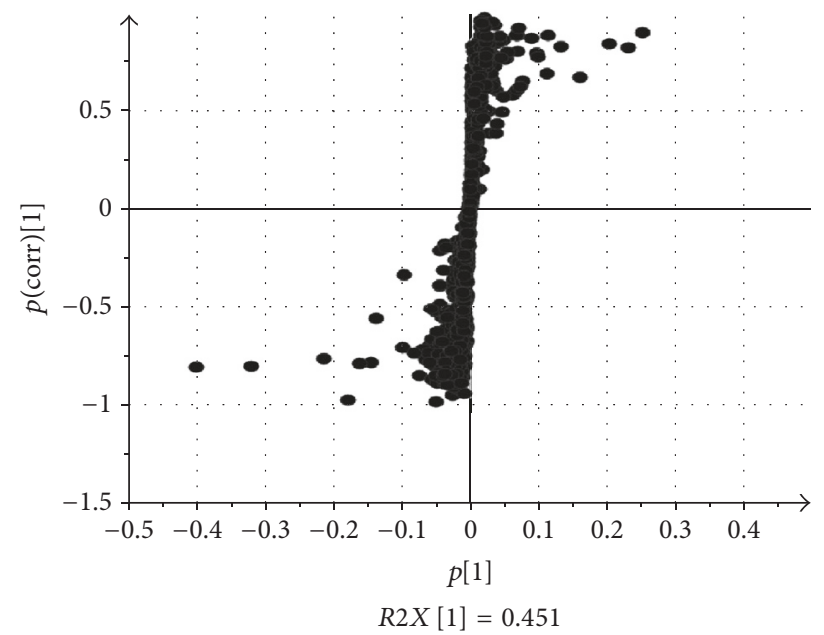

(c)

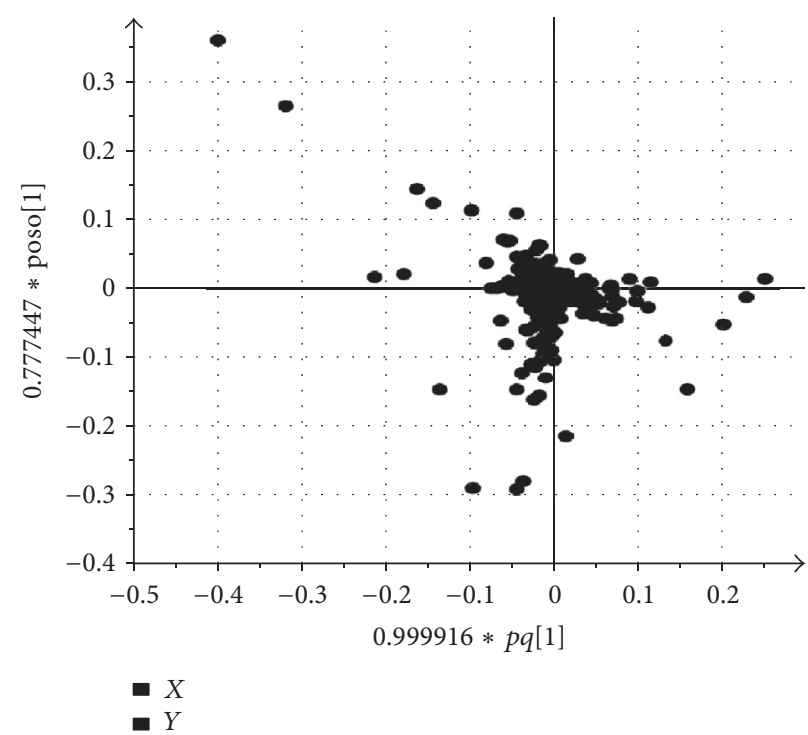

(b)

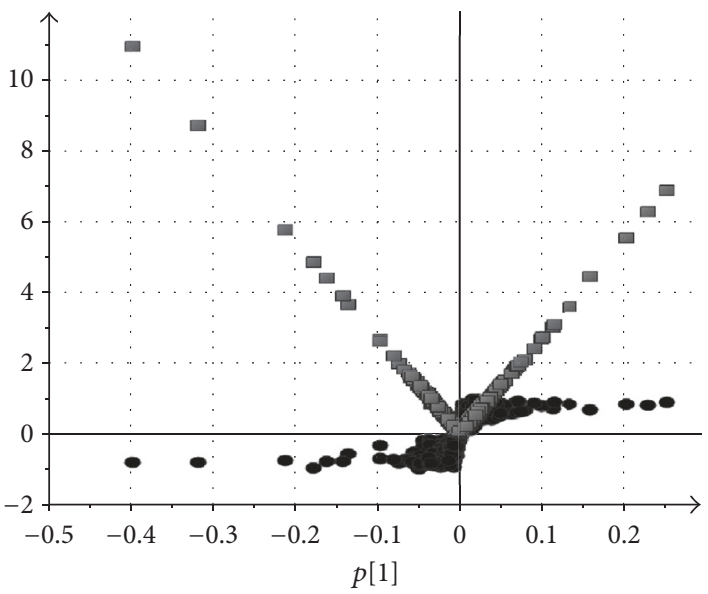

$R 2 X[1]=0.451$

- $p(\operatorname{corr})[1]$

- VIP[1]

(d)

FIGURE 5: Multivariate analysis of untargeted metabolomics data. (a) OPLS-DA score plots of serum metabolic profiling of model group (1) and Uncaria group (2). (b) Loading plots constructed from the supervised OPLS-DA. (c) S-plot constructed from the supervised OPLS-DA. (d) VIP-score plots constructed from the supervised OPLS-DA. The loading plot, S-plot, and VIP plot were carried out to select distinct variables as potential biomarkers.

Phosphoglyceride is one of the most abundant phospholipids in both animal and human body. It is the main component of biofilm, which participates in the signal recognition and transduction in cell membrane. Due to various substituent and replacement groups, phosphoglyceride could be divided into different classes, like phosphatidylcholine (PC), phosphatidylethanolamine (PE), and so on. PC was hydrolyzed by PLA2 from the sn-2 position, producing LysoPC and FFA. The generated endogenous LysoPC was the major active ingredient of oxidized low density lipoprotein (ox-LDL) [3]. LOX-1 receptor could be activated by ox-LDL and lead to dephosphorylation of serine residues in 1179/1177 site of eNOS. Then the activity of endothelial cell eNOS would be inhibited, and endothelium dependent relaxing function was suppressed. All of the above would lead to elevated blood pressure [35]. This confirmed that ox-LDL was an independent risk factor of hypertension. Besides, PLA2 was probably activated by free radicals $[36,37]$. In our study, PC was significantly decreased in the model group compared with that in the normal group, and LysoPC does the contrary (Figure 6). There is a tendency to return to normal after intervention by Uncaria. The results effectively indicated that 

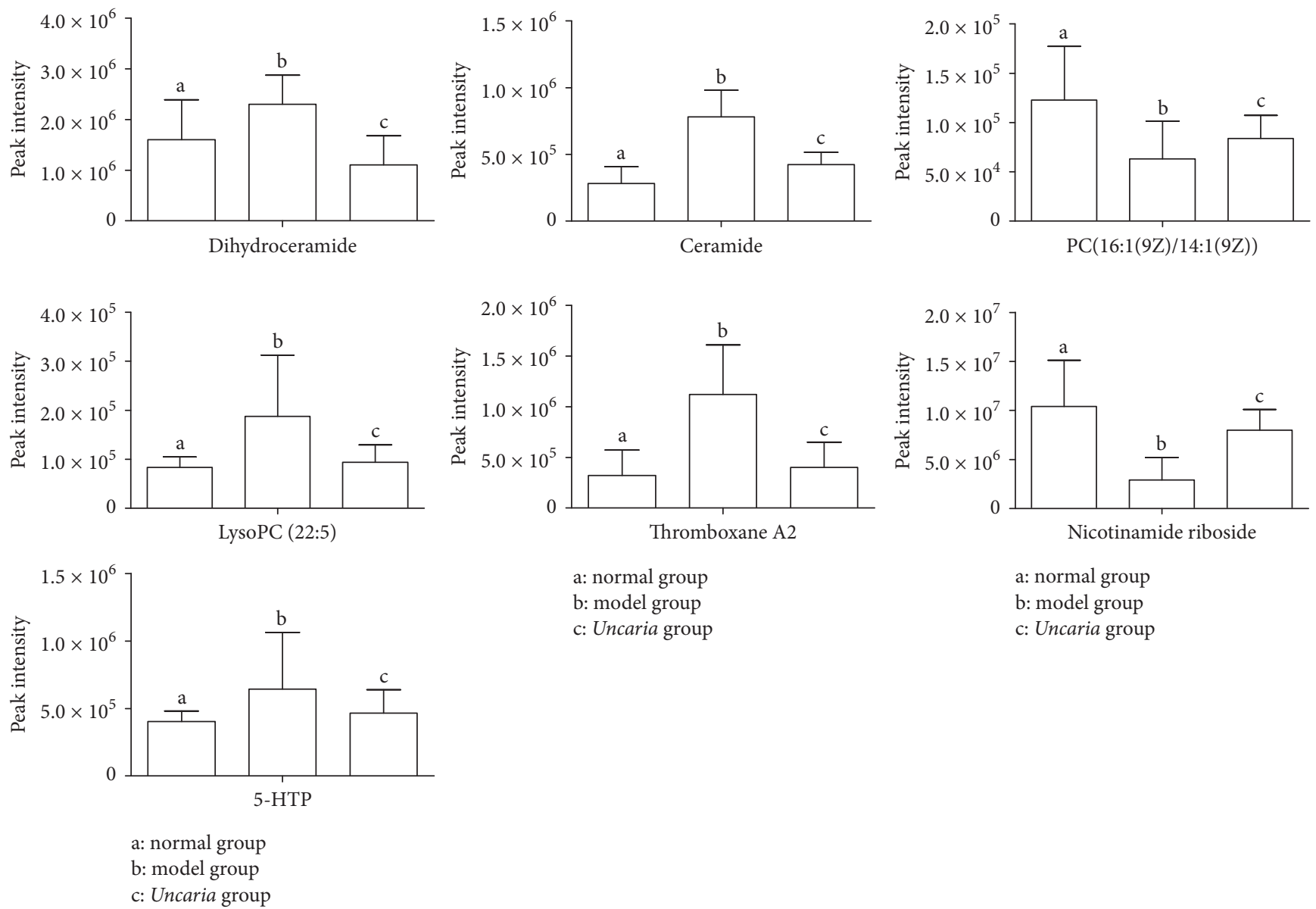

$$
\begin{aligned}
& \text { a: normal group } \\
& \text { b: model group } \\
& \text { c: Uncaria group }
\end{aligned}
$$

a: normal group

b: model group

c: Uncaria group

Figure 6: Comparison of different potential biomarkers of normal group, model group, and Uncaria-treated group. $p<0.05$ compared to model group.

Uncaria could correct phospholipid metabolism disorder to lower the blood pressure.

4.2. Vitamin and Amino Acids Metabolism. In addition to lipid metabolism disordered, the experiment results also find elevated level of nicotinamide ribose and reduced level of 5-HTP. Nicotinamide ribose, which is a component of $\mathrm{NADH}$ and $\mathrm{NADPH}$, is precursor of nicotinamide. It exerts protective effects against the oxidative stress process. With the increased level of NADPH, oxidase of generated ROS can be regulated, as well as maintaining the activity of antioxidant enzymes. Thus, the function of endothelial cells can be protected $[38,39]$. Furthermore, the activity of PLA2 could be inhibited by NADP to reduce the production of FFA and ceramide [40]. In present research, the variation of 5-HTP indicated that morbidity of hypertension may also be related to tryptophan metabolism. 5-HTP was produced from tryptophan by tryptophan hydroxylase and then transformed to decarboxylate 5-HT. Decarboxylate 5-HT is a potential vasoconstrictor and neuromodulator, which has strong effects in vessel contraction. As a result, 5-HTP was significantly decreased in the Uncaria-treated group in comparison with the model group (Figure 6). The reasons for above results may be as follows: firstly, the secretion of neurotransmitters such as norepinephrine could be reduced after Uncaria administration, which would inhibit excitation of central nervous system by decreasing level of 5-HT [41]. Secondly, as source of nicotinic acid and nicotinamide, 5HTP's activity of relevant molecules would be affected by tryptophan. Thus Uncaria may play a role in antihypertension through various pathways.

\section{Conclusion}

The serum metabolic profiling was detected and metabolomics was employed to demonstrate the intervention effects of Uncaria. In this paper, we observed a clear metabolic characteristic difference between the model group and Uncaria-treated groups. The level of 7 perturbed metabolites was reversed. The results suggested that the antihypertensive mechanism of Uncaria may involve regulating of lipid, vitamin, and amino acid metabolism, as well as being associated with vascular endothelial function. The results also indicated that Uncaria could be employed as a novel and effective antihypertensive treatment herb. In conclusion, metabolomics could give a holistic view of the 


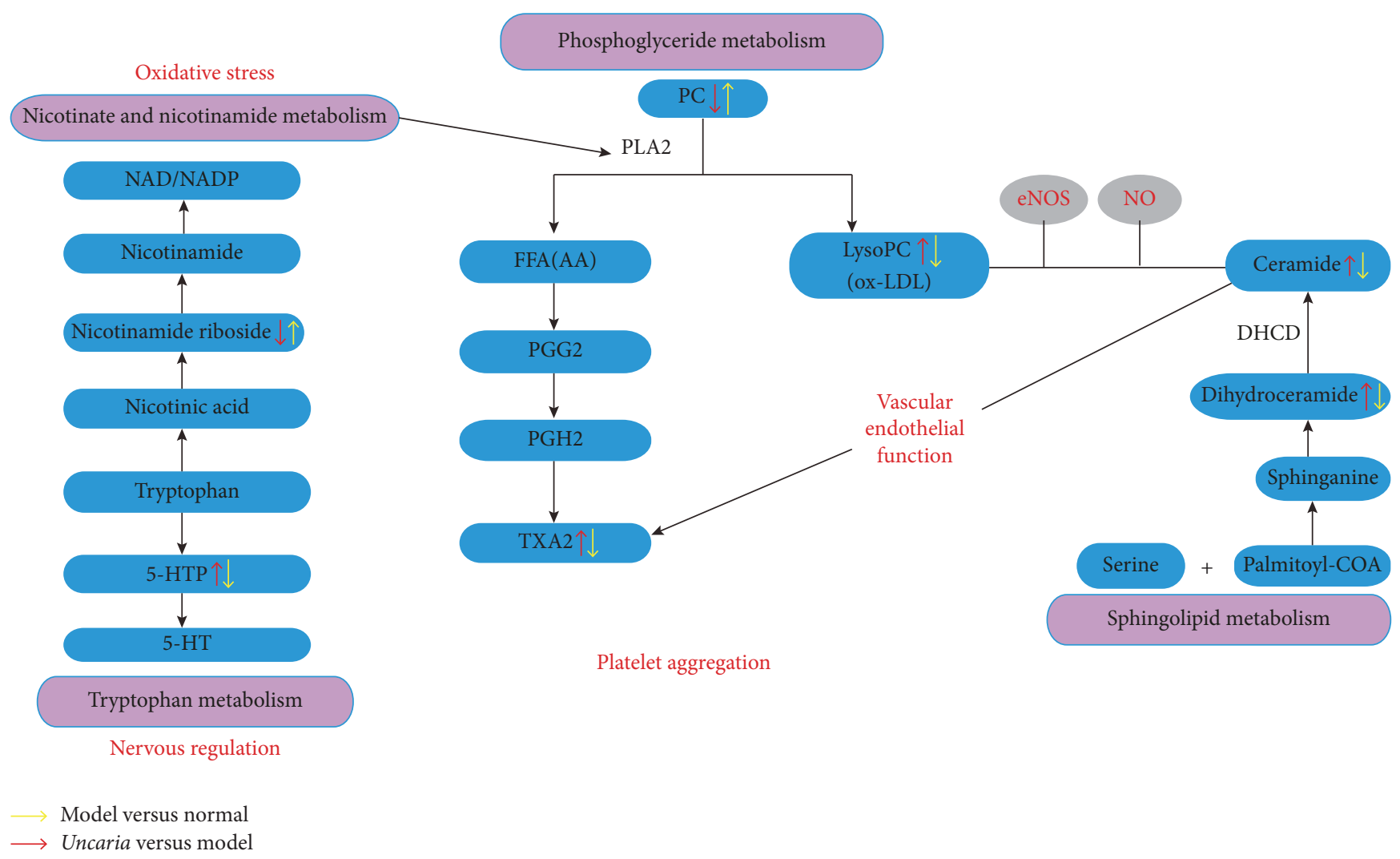

FIGURE 7: The network of the potential biomarkers variation for SHRs with or without Uncaria modulation.

disease pathogenesis and therapeutics; at the same time it might make great contributions to the evaluation of curative effects of TCM on hypertension.

\section{Conflicts of Interest}

The authors declare that there are no conflicts of interest regarding the publication of this paper.

\section{Authors' Contributions}

Yunlun Li and Haiqiang Jiang contributed equally to this work.

\section{Acknowledgments}

This study was financially supported by the foundation from the National Natural Science Foundation of China (no. 81102549, no. 81473653, and no. 81774173) and a project funded by China Postdoctoral Science Foundation (no. 2015T80741).

\section{Supplementary Materials}

Uncaria could be found in Chinese Pharmacopoeia 2015 version, but no active compounds were determined. According to the related reference, we determined four alkaloids including rhynchophylline, isorhynchophylline, corynoxeine, and isocorynoxeine by HPLC-QQQ-MS. Because the topic of the manuscript is metabolomics, it is shown here. (1) HPLCQQQ-MS/MS instrumentation and analytical conditions: Table a: parameters of LC-QQQ/MS/MS analysis for four constituents (alkaloids). Figure a: MRM chromatograms of four alkaloids in Uncaria extracts (chromatography of Uncaria extract). (2) Methodology: the precision, stability, repeatability, and recovery were also measured. Table b: linear equation, linear range, correlation coefficient, and detection limits of four constituents (alkaloids). Table c: the content of four constituents (alkaloids) in Uncaria extract. (Supplementary Materials)

\section{References}

[1] H. M. Perry Jr., J. P. Miller, J. R. Fornoff et al., "Early predictors of 15-year end-stage renal disease in hypertensive patients," Hypertension, vol. 25, no. 4 I, pp. 587-594, 1995.

[2] D. M. Bell, T. E. Johns, and L. M. Lopez, "Endothelial Dysfunction: Implications for Therapy of Cardiovascular Diseases," Annals of Pharmacotherapy, vol. 32, no. 4, pp. 459-470, 2016.

[3] S. M. L. Wallace, Yasmin, C. M. McEniery et al., "Isolated systolic hypertension is characterized by increased aortic stiffness and endothelial dysfunction," Hypertension, vol. 50, no. 1, pp. 228-233, 2007.

[4] H. Zhang and D. Cook, "Cerebral Vascular Smooth Muscle Potassium Channels and their Possible Role in the Management of Vasospasm," Pharmacology \& Toxicology, vol. 75, no. 6, pp. 327-336, 1994 
[5] C. G. C. Spencer, S. C. Martin, D. C. Felmeden, A. D. Blann, G. D. Beevers, and G. Y. H. Lip, "Relationship of homocysteine to markers of platelet and endothelial activation in 'high risk' hypertensives: A substudy of the Anglo-Scandinavian Cardiac Outcomes Trial," International Journal of Cardiology, vol. 94, no. 2-3, pp. 293-300, 2004.

[6] M. Fujita and T. Fujita, "The role of CNS in salt-sensitive hypertension," Current Hypertension Reports, vol. 15, no. 4, pp. 390-394, 2013.

[7] L. Feng, A. Manavalan, M. Mishra, S. K. Sze, J.-M. Hu, and K. Heese, "Tianma modulates blood vessel tonicity," The Open Biochemistry Journal, vol. 6, pp. 56-65, 2012.

[8] J. Wang, B. Feng, X. Yang et al., "Tianma Gouteng Yin as adjunctive treatment for essential hypertension: a systematic review of randomized controlled trials," Evidence-Based Complementary and Alternative Medicine, vol. 2013, Article ID 706125, 18 pages, 2013.

[9] A. Ndagijimana, X. Wang, G. Pan, F. Zhang, H. Feng, and O. Olaleye, "A review on indole alkaloids isolated from Uncaria rhynchophylla and their pharmacological studies," Fitoterapia, vol. 86, no. 1, pp. 35-47, 2013.

[10] Z. Yang and M. Qiu, "Cardiovascular effects of tanshinone II A and its mechanism research progress," Chinese Journal of Arteriosclerosis, vol. 19, no. 4, pp. 372-374, 2011.

[11] Y.-L. Li, "Effect of rhynchophylline and isorhynchophylline on the proliferation of rat vascular smooth muscle cell induced by angiotensin II," Chinese Pharmacological Bulletin, vol. 24, no. 1, pp. 53-58, 2008.

[12] J. K. Nicholson, J. C. Lindon, and E. Holmes, “"Metabonomics”: understanding the metabolic responses of living systems to pathophysiological stimuli via multivariate statistical analysis of biological NMR spectroscopic data," Xenobiotica, vol. 29, no. 11, pp. 1181-1189, 1999.

[13] D. Huang, J. Yang, X. Lu, Y. Deng, Z. Xiong, and F. Li, “An integrated plasma and urinary metabonomic study using UHPLCMS: intervention effects of Epimedium koreanum on 'KidneyYang Deficiency syndrome' rats," Journal of Pharmaceutical and Biomedical Analysis, vol. 76, pp. 200-206, 2013.

[14] J. C. Lindon, E. Holmes, and J. K. Nicholson, "Metabonomics and its role in drug development and disease diagnosis," Expert Review of Molecular Diagnostics, vol. 4, no. 2, pp. 189-199, 2004.

[15] H. C. Keun, "Metabonomic modeling of drug toxicity," Pharmacology \& Therapeutics, vol. 109, no. 1-2, pp. 92-106, 2006.

[16] D. B. Kell, "Systems biology, metabolic modelling and metabolomics in drug discovery and development," Drug Discovery Therapy, vol. 11, no. 23-24, pp. 1085-1092, 2006.

[17] L. Elh, V. K. Wong, Z. H. Jiang, and et al., "Integrated networkbased medicine: The role of traditional Chinese medicine in developing a new generation of medicine," Science, vol. 346, no. 6216 Suppl, p. 1569, 2014.

[18] L. X. Duan, T. L. Chen, M. Li et al., "Use of the metabolomics approach to characterize chinese medicinal material Huangqi," Molecular Plant, vol. 5, no. 2, pp. 376-386, 2012.

[19] H. Zhang, P. Fu, B. Ke et al., "Metabolomic analysis of biochemical changes in the plasma and urine of collagen-induced arthritis in rats after treatment with Huang-Lian-Jie-Du-Tang," Journal of Ethnopharmacology, vol. 154, no. 1, pp. 55-64, 2014.

[20] J. C. Lindon and J. K. Nicholson, "Spectroscopic and statistical techniques for information recovery in metabonomics and metabolomics," Annual Review of Analytical Chemistry, vol. 1, no. 1, pp. 45-69, 2008.
[21] J. L. Liu, H. L. Wang, L. F. Zhang et al., "Metabonomics study of brain-specific human S100B transgenic mice by using highperformance liquid chromatography coupled with quadrupole time of flight mass spectrometry," Biological \& Pharmaceutical Bulletin, vol. 34, no. 6, pp. 871-876, 2011.

[22] B. M. Psaty, C. D. Furberg, L. H. Kuller et al., "Association between blood pressure level and the risk of myocardial infarction, stroke, and total mortality: The cardiovascular health study," JAMA Internal Medicine, vol. 161, no. 9, pp. 1183-1192, 2001.

[23] D. S. Lee, J. M. Massaro, T. J. Wang et al., "Antecedent blood pressure, body mass index, and the risk of incident heart failure in later life," Hypertension, vol. 50, no. 5, pp. 869-876, 2007.

[24] R. Stewart, Q. Xue, K. Masaki et al., "Change in Blood Pressure and Incident Dementia: A 32-Year Prospective Study," Hypertension, vol. 54, no. 2, pp. 233-240, 2009.

[25] F. Lin, A. Manacle, M. Mishandle, and et al., "Titian modulates Blood Vessel Toni city," The Open Biochemistry Journal, vol. 6, pp. 56-65, 2012.

[26] L.-N. Tian, H.-W. Gao, Z.-J. Long, and L.-Q. Tang, "Antihypertensive effect and vascular regulation mechanism of rhynchophylline on spontaneously hypertensive rats," Chinese Traditional and Herbal Drugs, vol. 45, no. 15, pp. 2210-2213, 2014.

[27] M. I. Pörn-Ares, T. C. Saido, T. Andersson, and M. P. S. Ares, "Oxidized low-density lipoprotein induces calpain-dependent cell death and ubiquitination of caspase 3 in HMEC-1 endothelial cells," Biochemical Journal, vol. 374, no. 2, pp. 403-411, 2003.

[28] A. P. Wolffe and M. A. Matzke, "Epigenetics: regulation through repression," Science, vol. 286, no. 5439, pp. 481-486, 1999.

[29] H. Li, P. Junk, A. Huwiler et al., "Dual effect of ceramide on human endothelial cells: Induction of oxidative stress and transcriptional upregulation of endothelial nitric oxide synthase," Circulation, vol. 106, no. 17, pp. 2250-2256, 2002.

[30] L. Spijkers, R. Van den akker, B. Janssen et al., "Essential hypertension is associated with altered sphingolipid biology which can be normalised by anti-hypertensive treatment," Journal of Hypertension, vol. 29, p. e276, 2011.

[31] F. A. Kuehl Jr. and R. W. Egan, "Prostaglandins, arachidonic acid, and inflammation," Science, vol. 210, no. 4473, pp. 978-984, 1980.

[32] I. A. Jagroop, I. Clatworthy, J. Lewin, and D. P. Mikhailidis, "Shape change in human platelets: Measurement with a channelyzer and visualisation by electron microscopy," Platelets, vol. 11, no. 1, pp. 28-32, 2000.

[33] A. I. Schafer and R. I. Handin, "The role of platelets in thrombotic and vascular disease," Progress in Cardiovascular Diseases, vol. 22, no. 1, pp. 31-52, 1979.

[34] X.-L. Xie, M. Wu, Q. Wu, X.-N. Huang, Q.-H. Gong, and J.-S. Shi, "Inhibitory effect of Isorhynchophylline on platelet aggregation and thrombosis," Chinese Pharmacological Bulletin, vol. 23, no. 12, pp. 1636-1638, 2007.

[35] R. K. Davda, K. T. Stepniakowski, G. Lu, M. E. Ullian, T. L. Goodfriend, and B. M. Egan, "Oleic acid inhibits endothelial nitric oxide synthase by a protein kinase C-independent mechanism," Hypertension, vol. 26, no. 5, pp. 764-770, 1995.

[36] E. Takimoto and D. A. Kass, "Role of oxidative stress in cardiac hypertrophy and remodeling," Hypertension, vol. 49, no. 2, pp. 241-248, 2007.

[37] U. Wereszczyńska-Siemiaştkowska, A. Dą̧rowski, M. Jedynak, and A. Gabryelewicz, "Oxidative stress as an early prognostic 
factor in acute pancreatitis (AP): its correlation with serum phospholipase A2 (PLA2) and plasma polymorphonuclear elastase (PMN-E) in different-severity forms of human AP," Pancreas, vol. 17, no. 2, pp. 163-168, 1998.

[38] Z. Cao and Y. Li, "Chemical induction of cellular antioxidants affords marked protection against oxidative injury in vascular smooth muscle cells," Biochemical and Biophysical Research Communications, vol. 292, no. 1, pp. 50-57, 2002.

[39] J. Chen, W. Chen, M. Zhu, Y. Zhu, H. Yin, and Z. Tan, "Propofol attenuates angiotensin II-induced apoptosis in human coronary artery endothelial cells," British Journal of Anaesthesia, vol. 107, no. 4, pp. 525-532, 2011.

[40] L. Geeraert, G. P. Mannaerts, and P. P. Van Veldhoven, "Conversion of dihydroceramide into ceramide: Involvement of a desaturase," Biochemical Journal, vol. 327, no. 1, pp. 125-132, 1997.

[41] J.-Y. Zhou, Z.-X. Mo, and S.-W. Zhou, "Effect of rhynchophylline on central neurotransmitter levels in amphetamine-induced conditioned place preference rat brain," Fitoterapia, vol. 81, no. 7, pp. 844-848, 2010. 


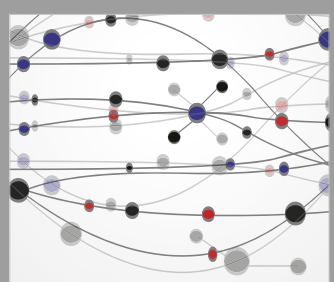

The Scientific World Journal
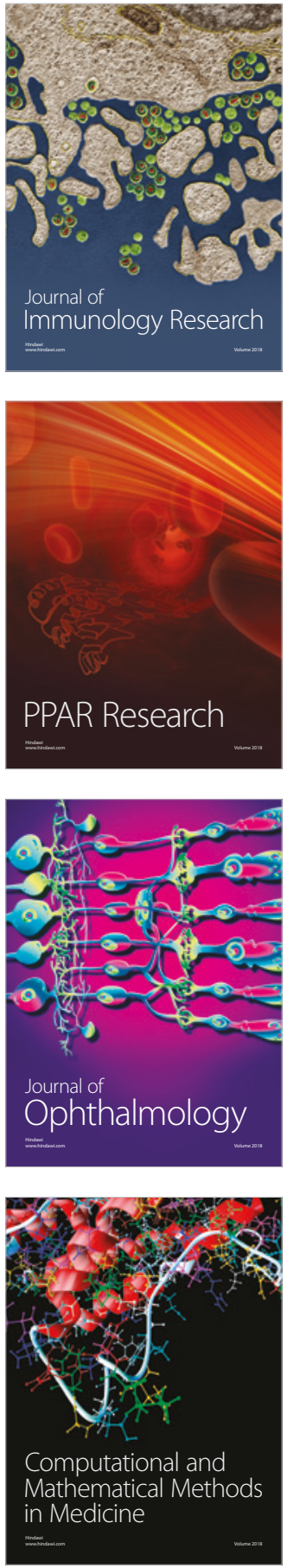

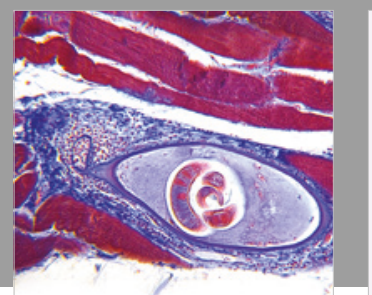

Gastroenterology Research and Practice

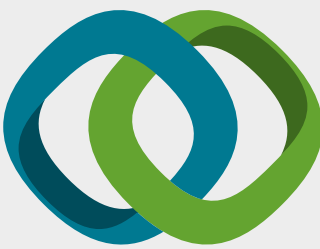

\section{Hindawi}

Submit your manuscripts at

www.hindawi.com
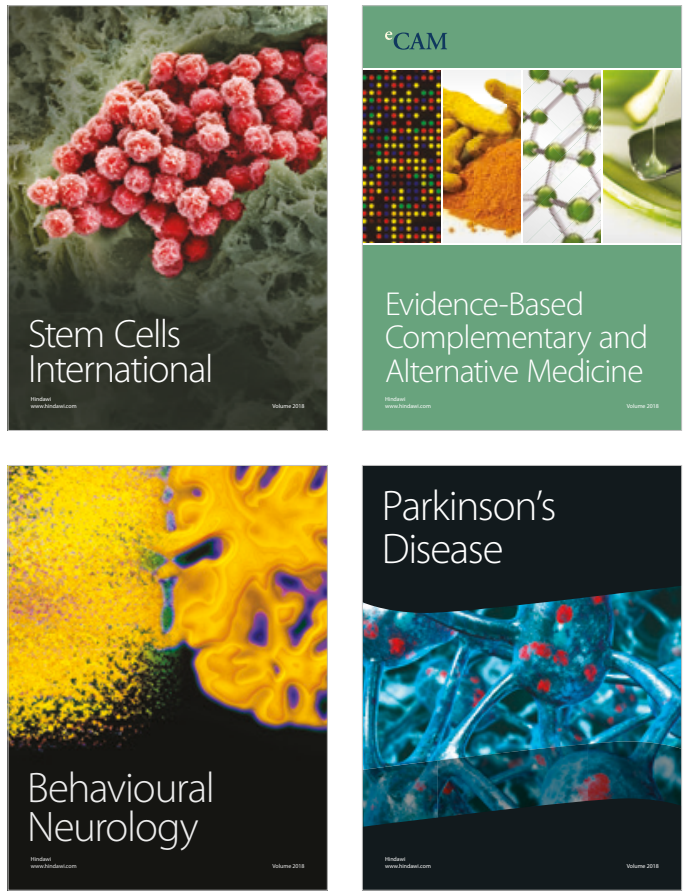

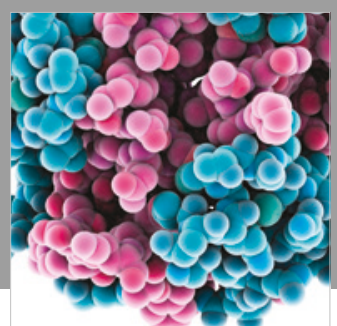

ournal of

Diabetes Research

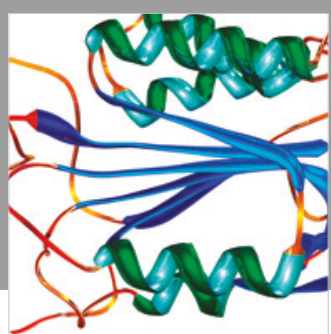

Disease Markers
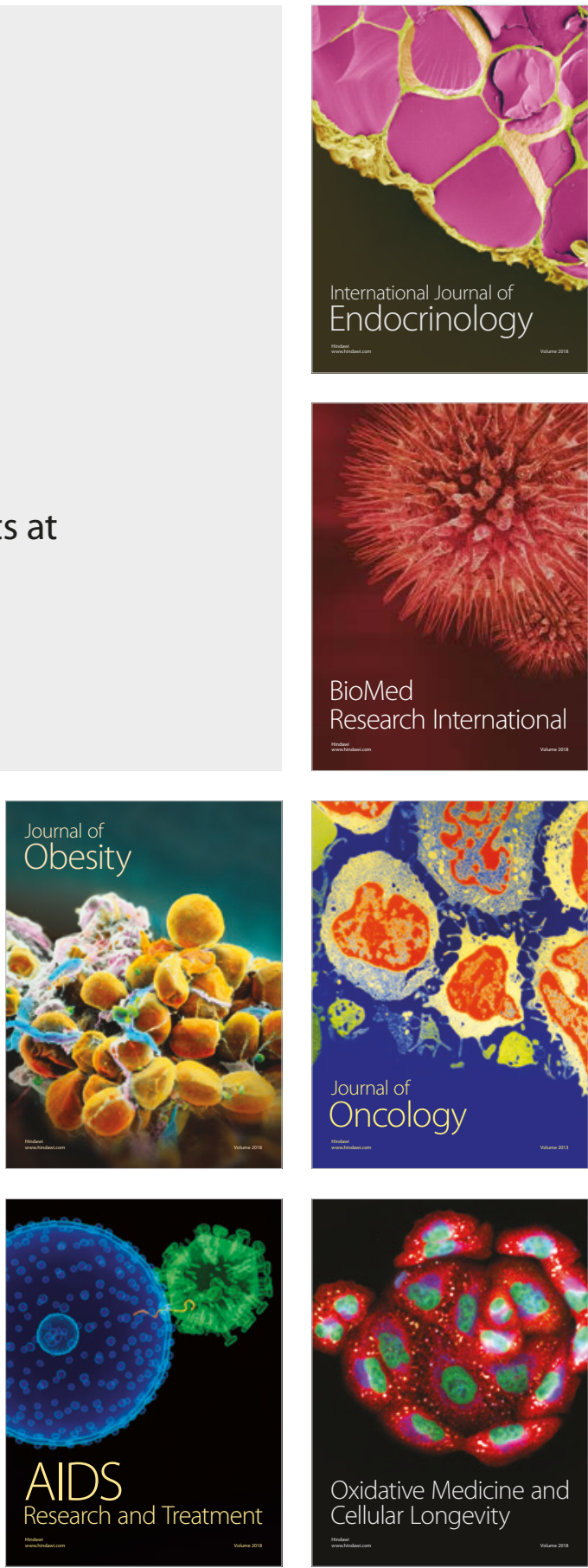